\title{
Development and characterization of microsatellite markers for diploid populations of the wind-pollinated herb Mercurialis annua
}

\author{
Ana Paula Machado, John R. Pannell and Jeanne Tonnabel ${ }^{*}$
}

\begin{abstract}
Objective: Mercurialis annua is a wind-pollinated annual plant that has long been used as a model for the study of ploidy and sexual-systems evolution. However, no molecular markers are yet available for genetic studies of its diploid populations. Here, we develop and characterize a set of eight polymorphic microsatellite markers for diploid dioecious M. annua.

Results: Following an SSR-enrichment protocol, 13 microsatellite markers were proposed, eight of which yielded successful amplification and polymorphism. We screened the eight microsatellite loci in 100 individuals. The number of alleles per marker ranged from 6 to 12, and observed heterozygosity ranged from 0.57 to 0.76 . To estimate potential allele scoring errors, these individuals' offspring were genotyped for the same loci, and error rates were estimated from parentage analyses. Error rates ranged from 0 to 6.8\%. Cross-amplification tests were performed for congeneric M. huetti and M. canariensis, with successful amplification for seven and six of the eight loci, respectively. The novel microsatellite markers proposed here will be crucial for a multitude of genetic studies of $M$. annua and further establish its importance as a model species for addressing ecological and population genetic questions.
\end{abstract}

Keywords: Population genetics, Phylogeography, Metapopulation dynamics, Microsatellites, Range expansion, Sexual systems, Dioecy

\section{Introduction}

Mercurialis annua is a wind-pollinated annual species that can be found in central and western Europe and around the Mediterranean Basin [1]. Across its distribution, $M$. annua displays remarkable variation in both ploidy, ranging from diploid to 12-ploid, and sexual system, ranging from dioecy to monoecy through androdioecy [1]. The species was first used as a model to address sex determination questions in the late 19th and early 20th centuries $[2,3]$. In the 1960 s, it became the focus of study of the evolution of ploidy and sexual systems [1]. More recently, M. annua has been used in studies of population range-expansion [e.g. 4] a topic that naturally requires the use of modern molecular genetic tools. For

\footnotetext{
*Correspondence: jeanne.tonnabel@unil.ch

†John R. Pannell and Jeanne Tonnabel are co-senior authors

Department of Ecology and Evolution, Biophore Building, University

of Lausanne, 1015 Lausanne, Switzerland
}

more information on the value of M. аnnua as a model system see [5] and references therein.

The scope for population-genetic and mating-system studies in diploid $M$. annua is currently limited by a lack of modern genetic markers. Indeed, the tools available are limited to isozyme loci [6], internal transcribed space (ITS) and chloroplast DNA sequences [7]. Although microsatellite markers have been successfully developed for the allohexaploid $M$. annua, these do not amplify in the diploid form [8]. Due to their high mutation rate and high polymorphism, microsatellites are powerful markers to detect genetic variation, allowing us to distinguish between closely related individuals, and thus to assess parentage and kinship relationships [9]. Additionally, it is a cost-effective PCR-based technique that does not require large quantities of DNA. As these qualities render microsatellites extremely advantageous genetic markers, we aimed to design a set of such markers that we could use in future work on diploid M. annua. Particularly, we 
are interested in studying selection gradients and kin selection in addition to metapopulation processes and population subdivision. Hence, we developed eight polymorphic microsatellite markers for diploid dioecious $M$. annua and examined cross-species amplification success in two other Mercurialis species.

\section{Main text}

Microsatellite markers were developed from DNA extractions of 15 individuals following an enrichment protocol at ecogenics $\mathrm{GmbH}$ (Balgach, Switzerland). Size-selected fragments from genomic DNA were enriched for simple sequence repeat (SSR) content using magnetic streptavidin beads and biotin-labelled CT and GT repeat oligonucleotides. The SSR-enriched library was analysed on an Illumina MiSeq platform using the Nano $2 \times 250$ v2 format. After assembly, 756 contigs/singlets contained a microsatellite insert with a tetra- or a trinucleotide of at least 6 repeat units or a dinucleotide of at least 10 repeat units. Of these, 465 contigs were suitable for primer design, pointing to the potential of 13 microsatellites markers with number of alleles ranging from 3 to 8. Individual tests for amplification and polymorphism were performed on 15 individuals, after which eight of the 13 potential markers were retained (Table 1). Markers of similar annealing temperature $\left(\mathrm{T}_{\mathrm{a}}\right)$ with different fluorescent tags and size ranges (Table 1) were grouped in three multiplexes. Primer3 [10] was used to avoid primer hybridisation within the multiplexes.

To characterize variation at the eight chosen loci, we genotyped 50 males and 50 females that had been sown as part of an outdoor common garden experiment at the field platform of the LabEx CeMEB in Montpellier (France). These plants originated from a mix of European populations encompassing the natural distribution of diploid M. апnиа. Total DNA was extracted using the BioSprint 96 DNA Plant Kit (Qiagen, Germany) following the manufacturer's instructions, and eluted in $100 \mu \mathrm{l}$ of distilled water. PCR amplification was carried out in a final volume of $10 \mu \mathrm{l}$, including $2 \times$ Multiplex PCR Master Mix (Qiagen, Germany), $1 \mu \mathrm{l}$ of extracted DNA, and variable primer concentrations (Table 1). Thermal cycling was performed in a TProfessional Standard Thermocycler (Biometra GmbH, Göttingen, Germany) as follows: $95{ }^{\circ} \mathrm{C}$ for $15 \mathrm{~min}$; 35 cycles of $94{ }^{\circ} \mathrm{C}$ for $30 \mathrm{~s}$, Ta (annealing temperature of each multiplex) for $90 \mathrm{~s}, 72{ }^{\circ} \mathrm{C}$ for $90 \mathrm{~s}$; and a final step at $72{ }^{\circ} \mathrm{C}$ for $10 \mathrm{~min}$. Diluted PCR products $(1 / 100)$ were analysed by capillary electrophoresis on an ABI3100 Genetic Analyzer (Applied Biosystems), with internal size standard GeneScan-350 LIZ. Fragment length analyses and scoring were performed with GeneMapper v4.0 (Applied Biosystems). Arlequin v3.5.2.2 [11] was used to calculate genetic diversity parameters, assess pairwise linkage disequilibrium

Table 1 Characteristics of the three primer multiplexes developed for M. annua, tested on 100 individuals

\begin{tabular}{|c|c|c|c|c|c|c|c|c|c|c|}
\hline$M$ & Locus & Primer sequences $\left(5^{\prime}-3^{\prime}\right)$ & Repeat motif & Size range (bp) & $\mathrm{Ta}\left({ }^{\circ} \mathrm{C}\right)$ & $\mathrm{FC}(\mu \mathrm{M})$ & $\mathrm{N}_{\mathrm{A}}$ & $H_{\mathrm{O}}$ & $H_{\mathrm{E}}$ & Error rate \% \\
\hline \multirow[t]{6}{*}{1} & 1101 & F 5'FAM-ACTAAAGTCCACTCATGGATGC & $(\mathrm{TCTT})_{9}$ & $94-115$ & 60 & 0.1 & 6 & 0.580 & 0.599 & 1.8 \\
\hline & & $\mathrm{R}$ GTATCCGTCCCTGGAGGAAG & & & & & & & & \\
\hline & 661 & F JOE-CAACGCTTTTGTACAGGGGG & $(\mathrm{CTAT})_{7}$ & $187-203$ & 60 & 0.1 & 6 & 0.620 & 0.685 & 0.0 \\
\hline & & R ACATTTATCCCTCCCCAGGC & & & & & & & & \\
\hline & 7575 & F AT550—AGTCACAGCTTGCAAATCTCC & $(\mathrm{TACA})_{7}$ & $222-255$ & 60 & 0.1 & 12 & 0.690 & 0.851 & 6.8 \\
\hline & & R TGCATCTTCTGGTTTCATCCC & & & & & & & & \\
\hline \multirow[t]{6}{*}{2} & 317 & F $\quad$ 5'FAM-TTTACCGAGATGCTCCCCAG & $(\mathrm{CTAT})_{7}$ & $188-273$ & 60 & 0.2 & 11 & 0.760 & 0.804 & 1.9 \\
\hline & & R AAAGCCCCTGGAATTGTTGC & & & & & & & & \\
\hline & 13654 & F JOE-TGCTGTTGGGATAAACAGGC & $(\mathrm{TCTT})_{9}$ & $191-219$ & 60 & 0.2 & 7 & 0.730 & 0.792 & 1.1 \\
\hline & & $\mathrm{R}$ CACTGCAAACGCATGAATCG & & & & & & & & \\
\hline & 4048 & F AT550-AGGGGTACCGTTTTGAGTCC & $(\mathrm{GT})_{12}$ & $212-240$ & 60 & 0.2 & 8 & 0.570 & 0.778 & 5.9 \\
\hline & & R CGATCCCTGCACAATCTGAC & & & & & & & & \\
\hline \multirow[t]{4}{*}{3} & 330 & F HEX-TAGCACGGAAACAAAAACGC & $(A A A G)_{8}$ & 168-196 & 59 & 0.2 & 9 & 0.670 & 0.769 & 0.8 \\
\hline & & R CTCGGTGGATTACCAAGACG & & & & & & & & \\
\hline & 3889 & F $\quad$ 5'FAM-TCGGTTTGATTTTTGAACACCC & $(\mathrm{TCTA})_{7}$ & $216-242$ & 59 & 0.2 & 7 & 0.710 & 0.696 & 0.0 \\
\hline & & $\mathrm{R}$ CACTGTAATCATGCCAAAGCTG & & & & & & & & \\
\hline
\end{tabular}

Fluorescent tags attached to the $5^{\prime}$ end of the forward primers and are indicated in italic

$\mathrm{M}$, multiplex; Ta, annealing temperature; $\mathrm{FC}$, final concentration of each set of primers in the PCR reaction; $\mathrm{N}_{\mathrm{A}}$, number of alleles; $H_{O}$, observed heterozygosity; $H_{\mathrm{E}}$ expected heterozygosity 
(LD) between loci and test for Hardy-Weinberg (HW) equilibrium.

To estimate the accuracy of allele scoring, 7-8 seeds per female (total of 391 seeds) were sown at the greenhouses of the Department of Ecology and Evolution of the University of Lausanne, Switzerland, and the resulting plants were genotyped for all markers. For each marker separately, we counted a mismatch when the offspring and its mother had no alleles in common. The error rate per marker was calculated as the proportion of mismatches across all offspring.

The number of alleles per locus ranged from 6 to 12 , the observed heterozygosity ranged from 0.57 to 0.76 , and the expected heterozygosity ranged from 0.60 to 0.85 (Table 1). Several markers showed significant linkage disequilibrium (LD) and deviations from Hardy-Weinberg (HW) equilibrium. Given that the tested samples were the offspring of the third generation of crosses within a small population, HW deviations are to be expected. For the same reason, the signal for LD is likely to reflect high relatedness between the individuals rather than physical linkage between the markers. Allelic frequencies across all markers ranged from 1 to $52.5 \%$. The error rate per marker ranged from 0 to $6.8 \%$ (Table 1).

Cross-amplification was checked in two sister species of $M$. annua: $M$. huetii (diploid) and $M$. canariensis (tetraploid). Five individuals of each sister species were genotyped for the three multiplexes in the same conditions as those described above, and similar genetic diversity calculations and tests for HW equilibrium were also performed. For the tetraploid $M$. canariensis, microsatellite scoring of alleles followed the gene dosage method, as described in [12], and genetic diversity parameters were calculated with AUTOTET [13].
The amplification in sister species was successful for most markers in both species, with the exception of locus 4048, which produced unspecific amplification and was therefore removed from the analyses, and locus 7575, which did not amplify in M. canariensis (Table 2). Loci 13654 and 661 were monomorphic for M. canariensis and $M$. huetii, respectively.

To our knowledge, no microsatellite loci have been developed for diploid M. апnиa to date (but note the existence of primers designed for allohexaploid $M$. annua [8]). The set of loci proposed here will be useful for further research in $M$. annua. In particular, studies of its range expansion, spatial population genetics, mating systems and paternity assignment will be greatly facilitated by these markers. The low error rates and the presence of rare alleles render them particularly useful for paternity analyses, which is of prime importance to understand several aspects of the evolution of sexual systems, including sexual selection, the evolution of sexual dimorphism and the estimation of fitness gain curves. Since most markers also amplified in the two congeneric species tested, our study broadens the perspectives of studies for these species too, as well as studies of homoploid and polyploid hybridization.

\section{Limitations}

- For some studies, eight markers may not be sufficient, for example to detect weak levels of structure within and between populations.

- The use of individuals from our common garden experiment after a few generations of crosses within a relatively small population may have yielded lower levels of polymorphism per marker than a wild population would.

Table 2 Cross-amplification of microsatellite markers in M. annua sister species

\begin{tabular}{|c|c|c|c|c|c|c|c|c|}
\hline \multirow[t]{2}{*}{ Locus } & \multicolumn{4}{|c|}{ M. canariensis (4n) } & \multicolumn{4}{|c|}{ M. huetii (2n) } \\
\hline & $\mathbf{N}_{\mathrm{A}}$ & Size Range(bp) & $H_{\mathrm{o}}$ & $H_{\mathrm{E}}$ & $\mathbf{N}_{\mathrm{A}}$ & Size range (bp) & $H_{0}$ & $H_{\mathrm{E}}$ \\
\hline 1101 & 3 & $94-111$ & 0.467 & 0.453 & 3 & $94-115$ & 1.000 & 0.644 \\
\hline 661 & 6 & $209-325$ & 0.567 & $0.742^{*}$ & 1 & 187 & - & - \\
\hline 7575 & 0 & - & - & - & 5 & $226-255$ & 0.200 & $0.867^{*}$ \\
\hline 317 & 8 & $196-216$ & 0.933 & 0.784 & 3 & $169-197$ & 1.000 & 0.689 \\
\hline 13654 & 1 & 184 & - & - & 3 & $184-215$ & 0.000 & $0.622^{*}$ \\
\hline 330 & 4 & $172-187$ & 0.567 & $0.583^{*}$ & 4 & $172-191$ & 0.800 & 0.733 \\
\hline 3889 & 3 & $230-242$ & 0.500 & 0.471 & 3 & $216-242$ & 0.600 & 0.689 \\
\hline
\end{tabular}

Locus 4048 is not shown as it did not amplify in any of the species. No significant linkage disequilibrium (LD) was found between loci

* Significant deviation from HW equilibrium ( $p$ value threshold at 0.0071 with Bonferroni correction) 


\section{Abbreviations}

SSR: simple sequence repeats; Ta: annealing temperature; PCR: polymerase chain reaction; LD: linkage disequilibrium; HW: Hardy-Weinberg.

\section{Authors' contributions}

JT and JRP designed the study. APM validated the markers on the sample set, performed cross-amplification tests, data analyses and initial interpretation of the results, and drafted the manuscript. JT tested the microsatellite markers and established the multiplexes. All authors read and approved the final manuscript.

\section{Acknowledgements}

We thank Guillaume Cossard for providing the M. annua plants for testing the markers; Paris Veltsos for providing M. huetti and M. canariensis samples for cross-amplification tests; and Nicolas Ruch for plant care.

\section{Competing interests}

The authors declare that they have no competing interests.

\section{Availability of data and materials}

Complete table with genotype data for all individuals and loci is available upon request to the corresponding author.

\section{Consent for publication}

Not applicable.

\section{Ethics approval and consent to participate}

Not applicable.

\section{Funding}

This work was funded by Grants to JRP from the Swiss National Science Foundation and the University of Lausanne, Switzerland.

\section{Publisher's Note}

Springer Nature remains neutral with regard to jurisdictional claims in published maps and institutional affiliations.

Received: 10 April 2017 Accepted: 29 July 2017

Published online: 10 August 2017

\section{References}

1. Le Durand B. Le complexe Mercurialis annua L. s.l.: une étude biosystématique. Ann Sci Nat Bot Paris. 1963:12:579-736.

2. Heyer F. Untersuchungen über das Verhältnis des Geschlechts bei einhäusigen und zweihäusigen Pflanzen, unter Berücksichtigung des Geschlechtsverhältnisses bei Thieren und Menschen. Berichte Physiologischen Laboratorium und der Versuchsanstalt des landwirtschaftlichen Instituts der Universität Halle. 1884; 1: 43

3. Yampolsky C. Inheritance of sex in Mercurialis annua. Am J Bot. 1919;6:410-42

4. Pujol B, Obbard DJ, Pannell JR. Symptoms of population range expansion: lessons from phenotypic and genetic differentiation in hexaploid Mercurialis annua. Plant Ecol Divers. 2010;3(2):103-8.

5. Pannell JR, Obbard DJ, Buggs RJA. Polyploidy and the sexual system: what can we learn from Mercurialis annua? Biol J Linn Soc. 2004;82(4):547-60.

6. Obbard DJ, Harris SA, Pannell JR. Sexual systems and population genetic structure in an annual plant: testing the metapopulation model. Am Nat. 2006:167(3):354-66.

7. Obbard DJ, Harris SA, Buggs RJA, Pannell JR. Hybridization, polyploidy, and the evolution of sexual systems in Mercurialis (Euphorbiaceae). Evolution. 2006;60(9):1801-15.

8. Korbecka G, Rymer PD, Harris SA, Pannell JR. Solving the problem of ambiguous paralogy for marker loci: microsatellite markers with diploid inheritance in allohexaploid Mercurialis annua (Euphorbiaceae). J Hered. 2010;101(4):504-11.

9. Hoshino AA, Bravo JP, Nobile PM, Morelli KA. Microsatellites as tools for genetic diversity analysis. In: Calistkan M, editor. Genetic diversity in microorganisms. Rijeka: InTech; 2012. p. 149-70.

10. Untergasser A, Cutcutache I, Koressaar T, Ye J, Faircloth BC, Remm B, Rozen SG. Primer3-new capabilities and interfaces. Nucleic Acids Res. 2012. doi:10.1093/nar/gks596.

11. Excoffier LG, Laval G, Schneider S. Arlequin (version 3.0): an integrated software package for population genetics data analysis. Evol Bioinform Online. 2005;1:47-50

12. Stöck M, Ustinova J, Lamatsch DK, Schartl M, Perrin N, Moritz C A vertebrate reproductive system involving three ploidy levels: hybrid origin of triploids in a contact zone of diploid and tetraploid palearctic green toads (Bufo viridis subgroup). Evolution. 2010 doi:10.1111/j.1558-5646.2009.00876.x.

13. Thrall $\mathrm{PH}$, Young A. AUTOTET: a program for analyses of autotetraploid genotypic data. J Hered. 2000;91(4):348-9.

\section{Submit your next manuscript to BioMed Central and we will help you at every step:}

- We accept pre-submission inquiries

- Our selector tool helps you to find the most relevant journal

- We provide round the clock customer support

- Convenient online submission

- Thorough peer review

- Inclusion in PubMed and all major indexing services

- Maximum visibility for your research

Submit your manuscript at www.biomedcentral.com/submit
Biomed Central 